\title{
Data Collection and Processing Methods for the Evaluation of Vehicle Road Departure Detection Systems
}

\author{
Dan Shen, Qiang Yi, Lingxi Li, Stanley Chien, Yaobin Chen, \\ Transportation Active Safety Institute \\ Indiana University-Purdue University Indianapolis, USA \\ Rini Sherony \\ Collaborative Safety Research Center (CSRC), \\ Toyota Motor North America, USA
}

\begin{abstract}
Road departure detection systems (RDDSs) for avoiding/mitigating road departure crashes have been developed and included on some production vehicles in recent years. In order to support and provide a standardized and objective performance evaluation of RDDSs, this paper describes the development of the data acquisition and data post-processing systems for testing RDDSs. Seven parameters are used to describe road departure test scenarios. The overall structure and specific components of data collection system and data post-processing system for evaluating vehicle RDDSs is devised and presented. Experimental results showed sensing system and data post-processing system could capture all needed signals and display vehicle motion profile from the testing vehicle accurately. Test track testing under different scenarios demonstrates the effective operations of the proposed data collection system.
\end{abstract}

\section{INTRODUCTION}

Vehicle crashes due to road departure is a leading cause of fatalities on US highways [1]. Approximately 12,000 drivers lose their lives each year due to road departure crashes [1]. Roadside crashes account for about 35 percent of the fatalities on nation's highways [2]. Road departure warning and road keeping assistance (RKA) systems are active safety technologies for dealing with this problem. Most of currently developed lane/road departure mitigation systems are based on the detection of lane markings. In addition, road departure detection systems mostly work on straight roads and slightly curved roads due to technological difficulties of road edge detection. However, many roads do not have lane markings or clear lane markings, especially in some rural and residential areas. Therefore, road departure detection and avoidance technologies could rely on the detection and identification of different types of road edges and road boundary objects on any kinds of road geometries.

Safety professionals and automobile manufacturers have strived to overcome the road departure issue by developing modeling methods, perception algorithms, and control strategies for road departure warning/mitigation systems. A simulated road departure detection system that relies on roadside terrain geometry analysis and subsequent threat assessment was discussed in [3]. The viability of detecting vehicle run-off the road through the measurements of anomalies under scenarios that left and right tires experience force imbalance was investigated in [4]. Authors in [5] explored effectiveness of a three-layer perceptron neural network to predict an unintentional road departure. A driving simulator testing that evaluates the road-departure prevention system in an emergency was presented in [6]. Authors in [7] proposed a system based on a closed-loop driver decision estimator (DDE), which determines the risk of road departure. Some other related research works include traffic forecast using deep learning method [8], evaluation of lane departure correction system (LDCS) based on the stochastic driver model [9], analysis of the LDCS utilizing naturalistic driving data [10], and so on.

Recently, more and more active safety technologies have been studied and developed on vehicle electronic stability control systems for advanced RDDSs. However, a fundamental question remains to be answered: "how to evaluate and demonstrate the effectiveness of the vehicle RDDSs on real roads?" Many testing and verification methods have been proposed based on virtual modeling and computer simulations [11-14]. However, comprehensive testing which combines virtual simulations and real-world testing is crucial. Therefore, the development of testing methods for vehicle road departure detection system is important. One such method is testing on a test track.

RDDSs on the market are only used on straight roads and slightly curved roads with clear lane markings. The primary goal and main contribution of this work is the development of a comprehensive method for testing vehicle road departure mitigation systems and for evaluating the road edge detection and control efficiency of RDDSs on all types of roads with/without lane markings. This approach includes test equipment development, and data collection and processing procedure, which provides a practical guidance on the development of next generation intelligent RDDSs with consideration.

The remainder of this paper is organized as follows. The key variables of road departure test scenarios based on

This is the author's manuscript of the article published in final edited form as:

Shen, D., Yi, Q., Li, L., Chien, S., Chen, Y., \& Sherony, R. (2018). Data Collection and Processing Methods for the Evaluation of Vehicle Road Departure Detection Systems. 2018 IEEE Intelligent Vehicles Symposium (IV), 1373-1378. 
representative national crash databases are discussed and determined in Section II. The overall structure for testing the vehicle road departure detection system is presented in Section III. Section IV describes the structure of data collection systems. Data post-processing system is presented in Section V. Finally, the conclusions and comments on the performance of the proposed method are given in Section VI.

\section{KEY PARAMETERS In ROAD DEPARTURE TESTING}

One question to be answered in road-departure mitigation system evaluation is what the representative testing conditions are. This question is addressed in this paper by focusing on the information and key parameters regarding the representative test scenarios and the vehicle parameter (departure speed vs. departure angle). The overall structure of road-departure evaluation system to be discussed in Section III is also based on the outcomes of key variables of road departure tests.

Since all test methods and results rely on road-departure test scenarios, the determination of key variables in these scenarios from national crash databases is crucial. To achieve this objective, an overall approach and process for road departure testing is proposed, as shown in Fig. 1. In this paper, we only describe how to determine the key variables for roaddeparture test scenarios. The determination of the most representative values for key parameters will be presented in a separate paper.

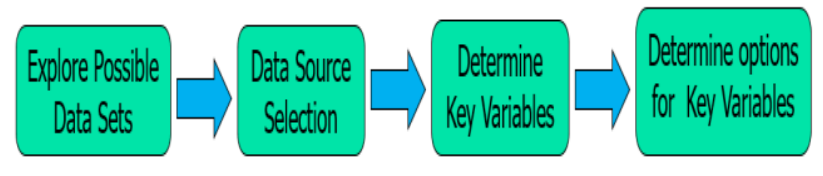

Fig. 1. Overall approach for road departure test scenarios.

Key parameters for road departure test scenarios can be obtained from the distribution of road departure conditions associated with run-off road crashes. These conditions contain variables including vehicle speed, road edge type, vehicle departure angle, and environmental factors. It is desirable that these conditions can be used to generate key parameters for RDDS testing. In this work, possible data sources containing conditions of road departure crashes were identified and analyzed. These data sources are from National Automotive Sampling System Crashworthiness Data System (NASS CDS), Fatality Analysis Reporting System (FARS), National Motor Vehicle Crash Causation Survey (NMVCCS), and so on.

In these databases, many factors/conditions are associated with the descriptions of road departure crashes. These factors are: (1) road conditions including road geometries, road surface, road slope, and radius of the curvature; (2) roadside conditions including road edges and boundaries; (3) vehicle conditions including vehicle departure speed, vehicle road departure angle, vehicle lateral speed, and side of road that the departure occurred; (4) environment conditions including weather, time, and lighting conditions; and (5) driver attentiveness such as distraction and fatigue. Since we are interested in the effectiveness of road-departure detection/mitigation systems, factor (5) is not applicable.
Therefore, seven key parameters for describing general roaddeparture test scenarios are selected as follows:

(1) Road type - the road geometries or alignment (straight road or curved road);

(2) Radius of road curvature - radius of curved road;

(3) Road edges type - roadside boundaries and materials, such as grass, gravel, concrete curb, metal guardrail, and so on;

(4) Vehicle departure velocity;

(5) Vehicle departure angle - the relationship between vehicle departure speed and vehicle departure angle are depicted in Fig. 2, where vehicle CG (center of gravity) velocity is the same as vehicle departure speed;

(6) Vehicle departure side - on which side of the road that road departure occurred; and

(7) Lighting conditions - time, weather condition, and street lighting.

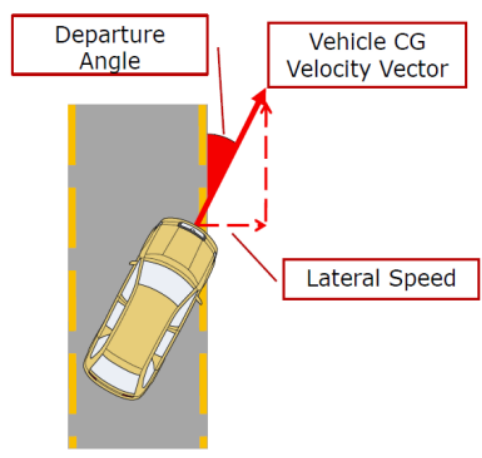

Fig. 2. Relationship between vehicle departure speed, lateral speed, and departure angle.

\section{STRUCTURE OF ROAD DEPARTURE TEST SYSTEM}

For the development of standardized performance evaluation of road-departure detection systems, the coordinated test data collection and post-processing systems were designed and implemented. Fig. 3 depicts the proposed overall structure for road departure detection system testing. The system includes: (1) a test vehicle with a data collection system, (2) surrogate roadside objects, including grass, metal guardrail, curb, and concrete divider, and, (3) a central

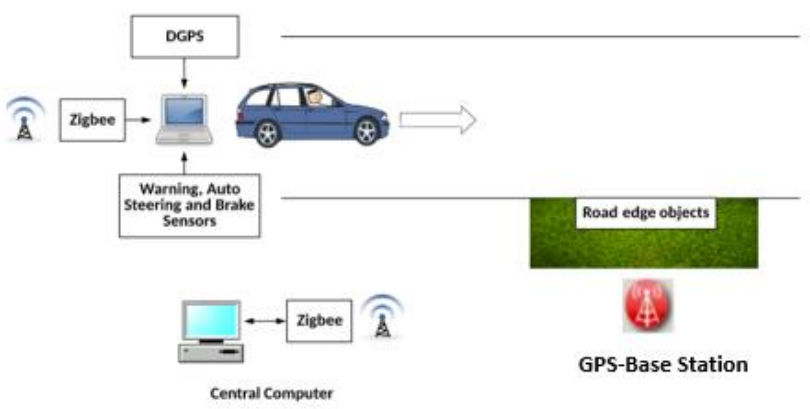

Fig. 3. Proposed structure of road departure testing system.

computer for data recording and system coordination and 2 
control. All components, except for the roadside surrogate objects, communicate with each other wirelessly through a ZigBee network with operation distance around one mile.

RDDS testing consists of two stages: data collection and post-data processing. A data acquisition system was developed to measure and collect all the required data during testing. The data post-processing system decodes, analyzes, plots, and displays test results.

\section{Data Collection System}

A vehicle road-departure detection system may have three action levels. The RDD system first gives a visual and/or audible warning to alert the driver to take corrective actions when vehicle is about to cross the boundary of the road. If the driver fails to take any action, the RDD system will actively apply the appropriate steering torque to push the vehicle back onto the road. If the vehicle cannot be pushed back and vehicle is on roadside, then autonomous braking might be applied.

For evaluating the performance of the road departure detection system, a data collection system is designed to capture and record the motion trajectory of the test vehicle, the activation time of vehicle's road departure detection actions (warning, auto-steering, and auto-braking), and behavior of the driver (steering and/or braking). The proposed structure of the data collection system is shown in Fig. 4. The data collection system consists of seven key components including sensor box, DGPS, in-vehicle computer, in-vehicle camera, CAN logger, vehicle speed display monitor, and OBD II interface.

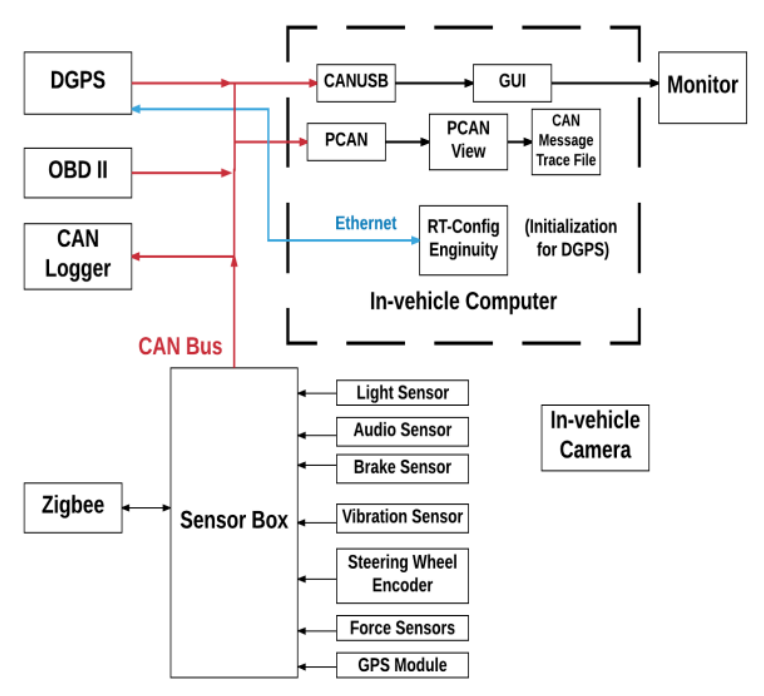

Fig. 4. Overall structure of the data collection system.

The DGPS is for measuring the motion profile and speed of the test vehicle. The sensor box is for capturing all visual, audible, and action signals generated by the vehicle, the driver, and the environment. These signals include brake pedal movement signal, brake light signals, warning sound, vehicle vibrations caused by crossing different road surfaces, steering wheel motion, and impact force from the bumper. The OBD II interface is used to query information from the test vehicle including vehicle longitudinal speed and engine speed. An invehicle camera is installed on the windshield to record the video data. The detailed introduction of these seven key components will be given in the following sub-sections.

All DGPS raw data are transferred through CAN bus to the in-vehicle computer and the CAN logger. The CAN logger records all messages in a SD memory card automatically. The raw CAN data is fed into two channels. One channel is a PCAN dongle connected to a PC. The data going through the PCAN dongle is processed by a PC software called 'PCAN view'. PCAN view saves the received data as trace files for post-processing. Another channel is a CAN USB dongle. The DGPS data going through the CAN USB dongle is processed by a software program to get the vehicle speed information (including departure speed, lateral speed, and departure angle). The vehicle speeds are shown on the PC monitor and an external monitor for the driver to compare the actual and desired vehicle speeds.

\section{A. Sensor Box}

The sensor box is developed to capture all signals from the test vehicle, including two light sensors, one audio sensor, one magnetic brake sensor, one vibration sensor, one steering angle and auto-steering motion detection encoder, several force sensors, and a time synchronized GPS module. The final assembled prototype of the sensor box is shown in Fig. 5.

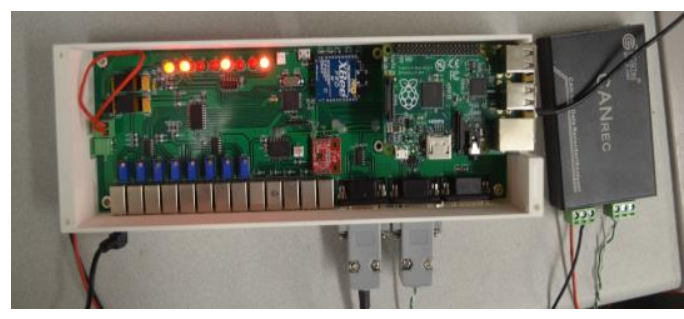

Fig. 5. Assembled prototype of sensor box.

The light sensors are designed to detect the road departure warning light on the dashboard (if there is any) when the road departure mitigation is activated, and the brake light when the driver presses the brake pedal or the road departure detection system automatically activates braking. The audio sensor is used to record the time and duration of warning sound when road departure detection is active. The magnetic brake sensor is designed to record brake pedal motion pressed by the driver. Combining with the brake light sensor and magnetic pedal brake sensors, we can figure out if braking is activated by the driver or the road-departure detection system. The encoder attached to the steering wheel is used to record the steering angle and detect the auto-steering motion. The force sensors are located on the front bumper of the testing vehicle. They are used to detect the time of impact and impact position on the vehicle. The GPS module is used to synchronize the signal time with DGPS system. The vibration sensor is a 3-axis acceleration sensor that is used to record the vibrations from the road surface and identify the rumble strips. 
The design objective of the sensor box is to receive all needed vehicle motion profile during road departure testing. The results are sent to both the CAN logger and in-vehicle computer through CAN bus. The ZigBee wireless communication module is also designed in the sensor box to exchange information with the central computer. The central computer is located outside of the test vehicle but close-by on the test track. The overall structure of the sensor box is shown in Fig. 6.

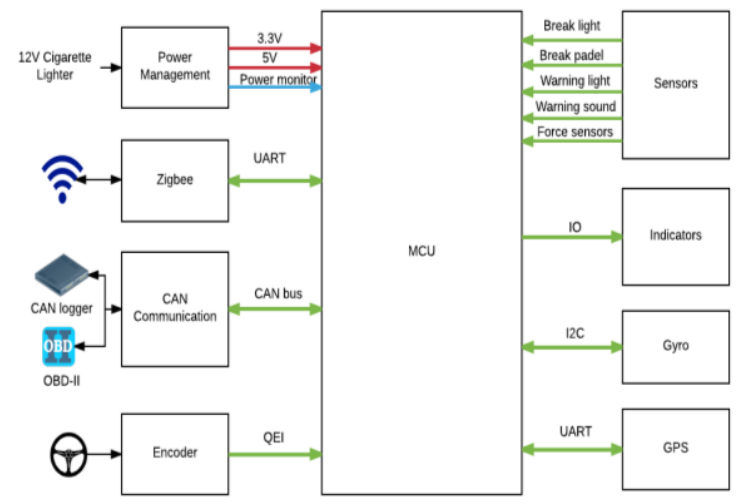

Fig. 6. Overall structure of the sensor box.

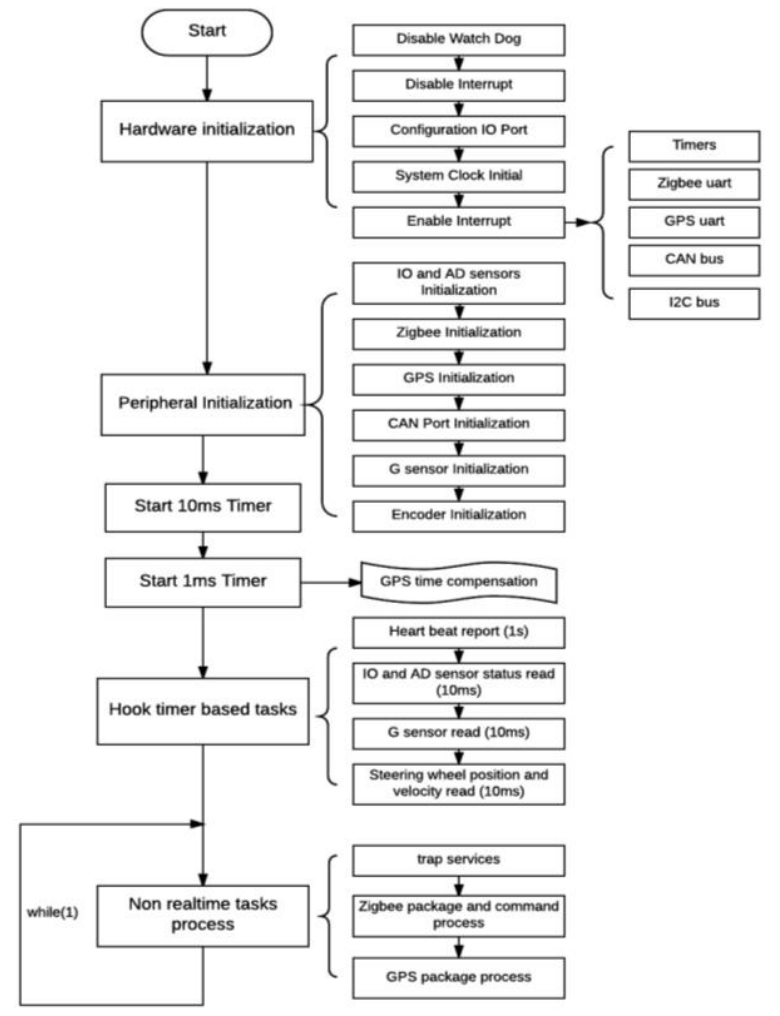

Fig. 7. Flow chart of sensor box operations

A foreground-background operating system is designed to allow the program in the sensor box to read the status of sensors, record data, synchronize GPS time, and communicate with laptop through CAN bus. The flow chart is shown in Fig.7. It should be mentioned that the audible warning signal from the vehicle is in a narrow frequency range. Thus, fast Fourier transform is implemented on a Raspberry Pi for audio signal processing. The Raspberry $\mathrm{Pi}$ is included in the sensor box to record vehicle road-departure warning beep in real time.

\section{B. Differential GPS (DGPS)}

The RT-3000 DGPS from OXTS is utilized to record test vehicle motion profile and motion speed. RT-3000 has four key components: in-vehicle RT-3000 unit, signal receiver, CAN connector, and GPS-base station. The GPS-base station is located on the test track; all other components are equipped on the vehicle. The finished installation is shown in Fig. 8.

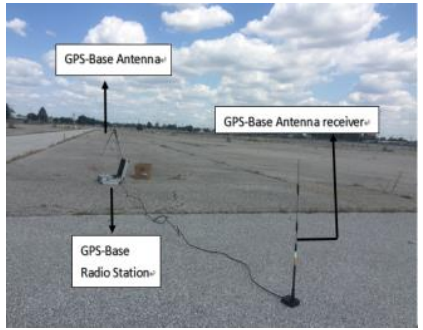

(a) Installed GPS-Base Station

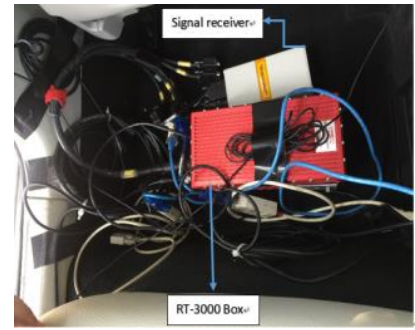

(b) In vehicle Components
Fig. 8. Installed DGPS components

\section{In-vehicle Computer}

A Windows based laptop PC is utilized to initialize the DGPS and record CAN messages. As shown in Fig. 4, the CAN data from the sensor box, DGPS are split into two channels. One channel goes through the PCAN dongle for raw data collection, the other channel goes through CAN-USB dongle for vehicle speed monitoring. PCAN and CAN-USB are two CAN to USB adapters (dongles) for receiving CAN messages.

A software PCAN-View developed by PEAK-System is installed in the in-vehicle computer. All raw data from CAN bus can be captured and saved as trace files to use for postprocessing. A GUI is developed based on CAN-USB to show the vehicle departure speed, vehicle lateral speed, and vehicle departure angle for helping the driver follow required vehicle forward and lateral speeds of test scenarios.

The vehicle forward speed, lateral speed, and departure angle are calculated for both straight road and curved road. For straight road, the above three key variables can be obtained directly by analyzing DGPS signal based on its local coordinates. For curved road, we drive and record a sequence of positions $\left(P_{y}, P_{x}\right)$ representing the boundary of a curved road. The second order fitting function can be generated to represent road boundary points. Then an algorithm is designed to compute the forward speed, lateral speed and departure angle according to local coordinates in DGPS. The first step of the algorithm for finding the closest point $\mathrm{D}$ to the moving vehicle A on a curved road is shown in Fig. 9. The second step of definition graph of the algorithm for computing vehicle motion profile is shown in Fig. 10.

\section{Other Components}

Other components in data collection system are in-vehicle video camera, CAN logger, and speed monitor. An in-vehicle camera is installed to record the video data during the testing 
information can be generated according to user's requirements. Some examples are:

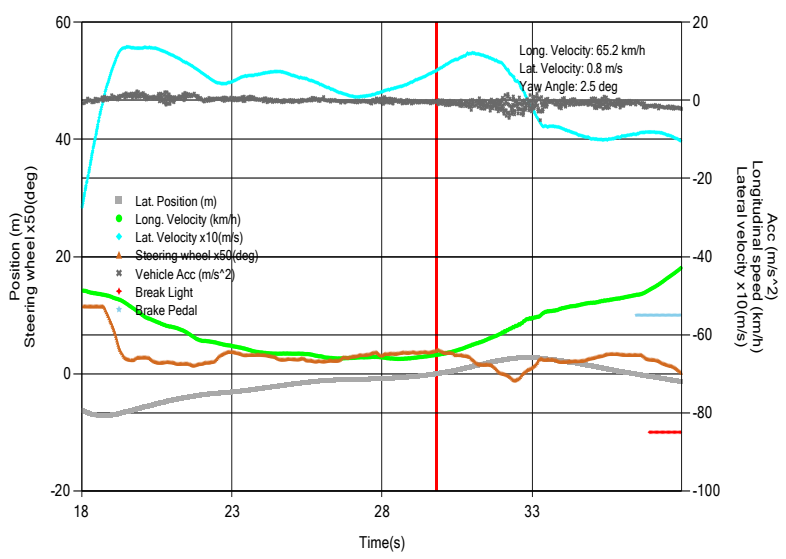

Fig. 13. Data post-processing for testing scenario 1

- Time from road departure warning to departure,

- Warning time to the auto-breaking,

- Time from warning to crash,

- Time from warning to the start of steering recovery,

- Time and position from the start of steering recovery to actual back to the road, and

- Minimum distance to the road edge.

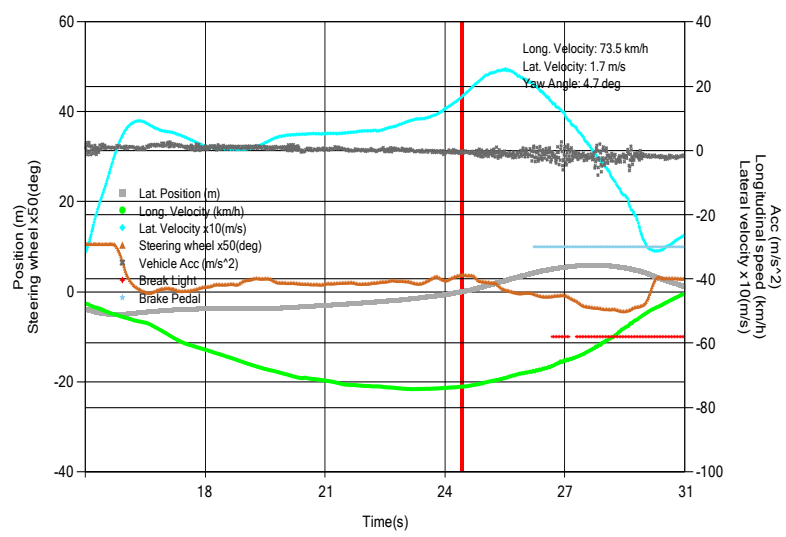

Fig. 14. Data post-processing for testing scenario 2

\section{CONCLUSIONS}

This paper has presented the development of a data collection system and data post-processing methods for vehicle road-departure detection systems on test track. The overall architecture of vehicle road departure testing system was proposed. Seven parameters for defining road departure scenarios were determined according to the most representative scenarios obtained from national crash data sources. These seven parameters are road type, radius of road curvature, road edges, vehicle departure velocity, vehicle departure angle, departure side, and lighting conditions. The integrated structure and detailed components of the data collection system were illustrated. The experiments demonstrated that the sensing system can record vehicle motion profile and capture all needed signals from the testing vehicle correctly. The algorithm for computing vehicle lateral speed and vehicle departure angle was also developed for both straight road and curved road. The data post-processing method was devised and implemented. The experimental results showed that the sensing system and data postprocessing system could accurately record all needed signals and display all vehicle motion profile from the test vehicle in different road departure scenarios.

\section{REFERENCES}

[1] National Academies of Sciences, Engineering, and Medicine. 2010. Identification of Vehicular Impact Conditions Associated with Serious Ran-off-Road Crashes. Washington, DC: The National Academies Press. https://doi.org/10.17226/14448.

[2] NCHRP 17-43. Long Term Roadside Crash Data Collection Program.2017.http://apps.trb.org/cmsfeed/TRBNetProjectDis play.asp?ProjectID=1637

[3] Arora, P., Corbin, D. and Brennan, S.N., 2016, June. Variablesensitivity road departure warning system based on static, mapped, near-road threats. In Intelligent Vehicles Symposium (IV), 2016 IEEE (pp. 1217-1223). IEEE.

[4] Yang, H., McBlane, D., Boyd, C., Beal, C. and Brennan, S., 2016, July. Vehicle road departure detection using anomalies in dynamics. In American Control Conference (ACC), 2016(pp. 6314-6319). IEEE.

[5] Ambarak, J.M., Ying, H., Syed, F. and Filev, D., 2017, March. A neural network for predicting unintentional lane departures. In Industrial Technology (ICIT), 2017 IEEE International Conference on (pp. 492-497). IEEE.

[6] Katzourakis, D.I., de Winter, J.C., Alirezaei, M., Corno, M. and Happee, R., 2014. Road-departure prevention in an emergency obstacle avoidance situation. IEEE transactions on systems, man, and cybernetics: systems, 44(5), pp.621-629.

[7] Y Alirezaei, M., Corno, M., Katzourakis, D., Ghaffari, A. and Kazemi, R., 2012. A robust steering assistance system for road departure avoidance. IEEE Transactions on Vehicular Technology, 61(5), pp.1953-1960.

[8] Zhao, Z., Chen, W., Wu, X., Chen, P.C. and Liu, J., 2017. LSTM network: a deep learning approach for short-term traffic forecast. IET Intelligent Transport Systems, 11(2), pp.68-75.

[9] Wang, W. and Zhao, D., 2017. Evaluation of Lane Departure Correction Systems Using a Stochastic Driver Model. arXiv preprint arXiv: 1702.05779 .

[10] Zhao, D., Wang, W. and LeBlanc, D.J., 2017. Evaluation of A Semi-Autonomous Lane Departure Correction System Using Naturalistic Driving Data. arXiv preprint arXiv:1702.06557.

[11] Ni, C., Gordon, T.J., Zhang, D. and Gao, Y., 2016, October. Test and validation of novel Lane-Departure Prevention System. In Students on Applied Engineering (ICSAE), International Conference for (pp. 441-446). IEEE.

[12] Xiong, G., Li, H., Ding, Z., Gong, J. and Chen, H., 2017, June. Subjective evaluation of vehicle active safety using PreScan and Simulink: Lane departure warning system as an example. In Vehicular Electronics and Safety (ICVES), 2017 IEEE International Conference on (pp. 208-213). IEEE.

[13] Tan, D., Chen, W. and Wang, H., 2017. On the Use of MonteCarlo Simulation and Deep Fourier Neural Network in Lane Departure Warning. IEEE Intelligent Transportation Systems Magazine, 9(4), pp.76-90.

[14] Wang, W. and Zhao, D., 2017. Evaluation of Lane Departure Correction Systems Using a Regenerative Stochastic Driver Model. IEEE Transactions on Intelligent Vehicles, 2(3), pp.221-232. 